\title{
Fuzzy Surface Slope Auto Tuner for Medical Robot
}

\author{
${ }^{1,2}$ Somayeh Jowkar, ${ }^{1}$ Farzin Piltan, ${ }^{1}$ Amirzubir Sahamijoo, ${ }^{1}$ Ali Taghizadegan, \\ ${ }^{1,3}$ Rouhollah Bahrami, ${ }^{1}$ Hossein Rashidi Bod, and ${ }^{1,4}$ Nasri. B Sulaiman \\ ${ }^{1}$ Intelligent System and Robotic Lab, Iranian Institute of Advanced Science and \\ Technology (IRAN SSP), Shiraz/Iran \\ ${ }^{2}$ Department of Information Technology, Faculty of Computer Engineering, \\ Ateneo De Manila University, Manila/Philippines \\ ${ }^{3}$ Department of Electrical-Electronic Engineering, Islamic Azad University, Iran \\ ${ }^{4}$ Department of Electrical Engineering, Faculty of Engineering, University Putra \\ Malaysia, Malaysia \\ Email:piltan_f@iranssp.org,www.iranssp.org/english
}

\begin{abstract}
Medical robots are sensitive tools to improve the surgery's performance. One of the most active research area in this field is control of medical robot. In this research, nonlinear, stable and robust Sliding Mode controller (SMC) is used as a based controller. This algorithm works based on the functional operation. The main traditional functions for this algorithm are switching and saturation functions. In this research, fuzzy algorithm is used to design a unique function to adjust the output performance. According to this research, the chattering eliminated based on applied modified sliding function, which is more robust than conventional sliding mode controller.
\end{abstract}

Keywords: surgical robots, four degrees of freedom, Sliding Mode Controller, fuzzy logic theory

\section{System's Dynamic (4 DOF Medical Robot Manipulator)}

Robot manipulators have many applications in aerospace, manufacturing, automotive, medicine and other industries. Robot manipulators consist of three main parts:

$\begin{array}{ll}\text { - } & \text { Mechanical } \\ \text { - } & \text { Electrical } \\ & \text { control. }\end{array}$

The dynamic functions are extracted from mechanical part of robot manipulator. A dynamic function is the study of motion with regard to the forces. Dynamic modeling of surgical robot manipulators is used to illustrate the behavior of robot manipulator (e.g., nonlinear dynamic behavior), design of nonlinear conventional controller and for simulation. It is used to analyses the relationship between dynamic functions output (e.g., joint motion, velocity, and accelerations) to input source of dynamic functions (e.g., force/torque or current/voltage). Dynamic functions is also used to explain the some dynamic parameter's effect (e.g., inertial matrix, Coriolios, Centrifugal, and some other parameters) to system's behavior [1].

The equation of multi degrees of freedom (DOF) surgical robot manipulator dynamics is considered by the following equation[1]:

$$
[A(\boldsymbol{q})] \ddot{\boldsymbol{q}}+[\boldsymbol{N}(\boldsymbol{q}, \dot{\boldsymbol{q}})]=[\tau]
$$

The dynamic formulations for 4 Degrees of Freedom serial links surgical robot manipulator are computed by; 


$$
A(\ddot{\theta})\left[\begin{array}{l}
\ddot{\theta}_{1} \\
\ddot{\theta}_{2} \\
\ddot{\theta}_{3} \\
\ddot{\theta}_{4}
\end{array}\right]+B(\theta)\left[\begin{array}{c}
\dot{\theta}_{1} \dot{\theta}_{2} \\
\dot{\theta}_{1} \dot{\theta}_{3} \\
\dot{\theta}_{1} \dot{\theta}_{4} \\
\dot{\theta}_{2} \dot{\theta}_{3} \\
\dot{\theta}_{2} \dot{\theta}_{4} \\
\dot{\theta}_{3} \dot{\theta}_{4}
\end{array}\right]+C(\theta)\left[\begin{array}{c}
\dot{\theta}_{1}^{2} \\
\dot{\theta}_{2}^{2} \\
\dot{\theta}_{3}^{2} \\
\dot{\theta}_{4}^{2}
\end{array}\right]+G(\theta)=\left[\begin{array}{l}
\tau_{1} \\
\tau_{2} \\
\tau_{3} \\
\tau_{4}
\end{array}\right]
$$

Where

$$
A(q)=\left[\begin{array}{cccc}
A_{11} & A_{12} & A_{13} & 0 \\
A_{21} & A_{22} & A_{23} & 0 \\
A_{31} & A_{32} & A_{33} & 0 \\
0 & 0 & 0 & A_{44}
\end{array}\right]
$$

According to [8] the inertial matrix elements $(\boldsymbol{A})$ are

$$
\begin{gathered}
A_{11}=I_{m 1}+I_{1}+I_{3} \times \cos \left(\theta_{2}\right) \cos \left(\theta_{2}\right)+I_{7} \sin \left(\theta_{2}+\theta_{3}\right) \sin \left(\theta_{2}+\theta_{3}\right) \\
+I_{10} \sin \left(\theta_{2}+\theta_{3}\right) \cos \left(\theta_{2}+\theta_{3}\right)+I_{11} \sin \left(\theta_{2}\right) \cos \left(\theta_{2}\right) \\
+I_{21} \sin \left(\theta_{2}+\theta_{3}\right) \sin \left(\theta_{2}+\theta_{3}\right)+2+\left[I_{5} \cos \left(\theta_{2}\right) \sin \left(\theta_{2}+\theta_{3}\right)\right. \\
+I_{12} \cos \left(\theta_{2}\right) \cos \left(\theta_{2}+\theta_{3}\right)+I_{15} \sin \left(\theta_{2}+\theta_{3}\right) \sin \left(\theta_{2}+\theta_{3}\right) \\
+I_{16} \cos \left(\theta_{2}\right) \sin \left(\theta_{2}+\theta_{3}\right)+I_{22} \sin \left(\theta_{2}+\theta_{3}\right) \cos \left(\theta_{2}+\theta_{3}\right) \\
A_{12}=I_{4} \sin \left(\theta_{2}\right)+I_{8} \cos \left(\theta_{2}+\theta_{3}\right)+I_{9} \cos \left(\theta_{2}\right)+I_{13} \sin \left(\theta_{2}+\theta_{3}\right)-I_{18} \cos \left(\theta_{2}+\theta_{3}\right) \\
A_{13}=I_{8} \cos \left(\theta_{2}+\theta_{3}\right)+I_{13} \sin \left(\theta_{2}+\theta_{3}\right)-I_{18} \cos \left(\theta_{2}+\theta_{3}\right) \\
A_{22}=I_{m 2}+I_{2}+I_{6}+2\left[I_{5} \sin \left(\theta_{3}\right)+I_{12} \cos \left(\theta_{2}\right)+I_{15}+I_{16} \sin \left(\theta_{3}\right)\right. \\
A_{23}=I_{5} \sin \left(\theta_{3}\right)+I_{6}+I_{12} \cos \left(\theta_{3}\right)+I_{16} \sin \left(\theta_{3}\right)+2 I_{15} \\
A_{33}=I_{m 3}+I_{6}+2 I_{15} \\
A_{44}=I_{m 4}+I_{14} \\
A_{21}=A_{12}, A_{31}=A_{13} \text { and } A_{32}=A_{23}
\end{gathered}
$$

The Corilios $(b)$ matrix elements are;

$$
b(q)=\left[\begin{array}{cccc}
b_{112} & b_{113} & 0 & b_{123} \\
0 & 0 & b_{214} & b_{223} \\
0 & 0 & b_{314} & 0 \\
b_{412} & b_{413} & 0 & 0
\end{array}\right]
$$

Where,

$$
\begin{aligned}
b_{112}=2\left[-I_{3}\right. & \sin \left(\theta_{2}\right) \cos \left(\theta_{2}\right)+I_{5} \cos \left(\theta_{2}+\theta_{2}+\theta_{3}\right)+I_{7} \sin \left(\theta_{2}+\theta_{3}\right) \cos \left(\theta_{2}+\theta_{3}\right) \\
& -I_{12} \sin \left(\theta_{2}+\theta_{2}+\theta_{3}\right)-I_{15} 2 \sin \left(\theta_{2}+\theta_{3}\right) \cos \left(\theta_{2}+\theta_{3}\right) \\
& +I_{16} \cos \left(\theta_{2}+\theta_{2}+\theta_{3}\right)+I_{21} \sin \left(\theta_{2}+\theta_{3}\right) \cos \left(\theta_{2}+\theta_{3}\right) \\
& \left.+I_{22}\left(1-2 \sin \left(\theta_{2}+\theta_{3}\right) \sin \left(\theta_{2}+\theta_{3}\right)\right)\right] \\
& +I_{10}\left(1-2 \sin \left(\theta_{2}+\theta_{3}\right) \sin \left(\theta_{2}+\theta_{3}\right)\right)+I_{11}\left(1-2 \sin \left(\theta_{2}\right) \sin \left(\theta_{2}\right)\right)
\end{aligned}
$$




$$
\begin{gathered}
b_{113}=2\left[I_{5} \cos \left(\theta_{2}\right) \cos \left(\theta_{2}+\theta_{3}\right)+I_{7} \sin \left(\theta_{2}+\theta_{3}\right) \cos \left(\theta_{2}+\theta_{3}\right)\right. \\
-I_{12} \cos \left(\theta_{2}\right) \sin \left(\theta_{2}+\theta_{2}\right)+I_{15} 2 \sin \left(\theta_{2}+\theta_{3}\right) \cos \left(\theta_{2}+\theta_{3}\right) \\
+I_{16} \cos \left(\theta_{2}\right) \cos \left(\theta_{2}+\theta_{3}\right)+I_{21} \sin \left(\theta_{2}+\theta_{3}\right) \cos \left(\theta_{2}+\theta_{3}\right) \\
\left.+I_{22}\left(1-2 \sin \left(\theta_{2}+\theta_{3}\right) \sin \left(\theta_{2}+\theta_{3}\right)\right)\right] \\
+I_{10}\left(1-2 \sin \left(\theta_{2}+\theta_{3}\right) \sin \left(\theta_{2}+\theta_{3}\right)\right) \\
b_{123}=2[ \\
\left.-I_{8} \sin \left(\theta_{2}+\theta_{3}\right)+I_{13} \cos \left(\theta_{2}+\theta_{3}\right)+I_{18} \sin \left(\theta_{2}+\theta_{3}\right)\right] \\
b_{214}=I_{14} \sin \left(\theta_{2}+\theta_{3}\right)+I_{19} \sin \left(\theta_{2}+\theta_{3}\right)+2 I_{20} \sin \left(\theta_{2}+\theta_{3}\right)(1-0.5) \\
b_{223}=2\left[-I_{12} \sin \left(\theta_{3}\right)+I_{5} \cos \left(\theta_{3}\right)+I_{16} \cos \left(\theta_{3}\right)\right] \\
b_{314}=2\left[I_{20} \sin \left(\theta_{2}+\theta_{3}\right)(1-0.5)\right]+I_{14} \sin \left(\theta_{2}+\theta_{3}\right)+I_{19} \sin \left(\theta_{2}+\theta_{3}\right) \\
b_{412}=b_{214}=-\left[I_{14} \sin \left(\theta_{2}+\theta_{3}\right)+I_{19} \sin \left(\theta_{2}+\theta_{3}\right)+2 I_{20} \sin \left(\theta_{2}+\theta_{3}\right)(1-0.5)\right] \\
b_{413}=-b_{314}=-2\left[I_{20} \sin \left(\theta_{2}+\theta_{3}\right)(1-0.5)\right]+I_{14} \sin \left(\theta_{2}+\theta_{3}\right)+I_{19} \sin \left(\theta_{2}+\theta_{3}\right)
\end{gathered}
$$

Based on above discussion $[b(q)]$ is $4 \times 6$ matrix and $[\dot{q} \dot{q}]$ is $6 \times 1$, therefore $[b(q) \cdot \dot{q} \dot{q}]$ is $4 \times 1$.

$$
[b(q) \cdot \dot{q} \dot{q}]_{4 \times 1}=\left[\begin{array}{c}
b_{112} \cdot q_{1} q_{2}+b_{113} \cdot q_{1} q_{3}+0+b_{123} \cdot q_{2} q_{3} \\
0+b_{214} \cdot q_{1} q_{4}+b_{223} \cdot q_{2} q_{3} \\
b_{314} \cdot q_{1} q_{4} \\
b_{412} \cdot q_{1} q_{2}+b_{413} \cdot q_{1} q_{3}
\end{array}\right]
$$

According to [8] Centrifugal $(C)$ matrix elements are;

$$
C(q)=\left[\begin{array}{cccc}
0 & C_{12} & C_{13} & 0 \\
C_{21} & C_{22} & C_{23} & 0 \\
C_{31} & C_{32} & 0 & 0 \\
0 & 0 & 0 & 0
\end{array}\right]
$$

Where,

$$
\begin{aligned}
& c_{12}=I_{4} \cos \left(\theta_{2}\right)-I_{8} \sin \left(\theta_{2}+\theta_{3}\right)-I_{9} \sin \left(\theta_{2}\right)+I_{13} \cos \left(\theta_{2}+\theta_{3}\right)+I_{18} \sin \left(\theta_{2}+\theta_{3}\right) \\
& c_{13}=0.5 b_{123}=-I_{8} \sin \left(\theta_{2}+\theta_{3}\right)+I_{13} \cos \left(\theta_{2}+\theta_{3}\right)+I_{18} \sin \left(\theta_{2}+\theta_{3}\right) \\
& c_{21}=-0.5 b_{112}=I_{3} \sin \left(\theta_{2}\right) \cos \left(\theta_{2}\right)-I_{5} \cos \left(\theta_{2}+\theta_{2}+\theta_{3}\right) \\
& -I_{7} \sin \left(\theta_{2}+\theta_{3}\right) \cos \left(\theta_{2}+\theta_{3}\right)+I_{12} \sin \left(\theta_{2}+\theta_{2}+\theta_{3}\right) \\
& +I_{15} 2 \sin \left(\theta_{2}+\theta_{3}\right) \cos \left(\theta_{2}+\theta_{3}\right)-I_{16} \cos \left(\theta_{2}+\theta_{2}+\theta_{3}\right) \\
& -I_{21} \sin \left(\theta_{2}+\theta_{3}\right) \cos \left(\theta_{2}+\theta_{3}\right) \\
& -I_{22}\left(1-2 \sin \left(\theta_{2}+\theta_{3}\right) \sin \left(\theta_{2}+\theta_{3}\right)\right) \\
& -0.5 I_{10}\left(1-2 \sin \left(\theta_{2}+\theta_{3}\right) \sin \left(\theta_{2}+\theta_{3}\right)\right) \\
& -0.5 I_{11}\left(1-2 \sin \left(\theta_{2}\right) \sin \left(\theta_{2}\right)\right)
\end{aligned}
$$




$$
\begin{aligned}
& c_{22}=0.5 b_{223}=-I_{12} \sin \left(\theta_{3}\right)+I_{5} \cos \left(\theta_{3}\right)+I_{16} \cos \left(\theta_{3}\right) \\
& \begin{aligned}
c_{23}=-0.5 b_{113} & =-I_{5} \cos \left(\theta_{2}\right) \cos \left(\theta_{2}+\theta_{3}\right)-I_{7} \sin \left(\theta_{2}+\theta_{3}\right) \cos \left(\theta_{2}+\theta_{3}\right) \\
& +I_{12} \cos \left(\theta_{2}\right) \sin \left(\theta_{2}+\theta_{2}\right)-I_{15} 2 \sin \left(\theta_{2}+\theta_{3}\right) \cos \left(\theta_{2}+\theta_{3}\right) \\
& -I_{16} \cos \left(\theta_{2}\right) \cos \left(\theta_{2}+\theta_{3}\right)-I_{21} \sin \left(\theta_{2}+\theta_{3}\right) \cos \left(\theta_{2}+\theta_{3}\right) \\
& -I_{22}\left(1-2 \sin \left(\theta_{2}+\theta_{3}\right) \sin \left(\theta_{2}+\theta_{3}\right)\right) \\
- & 0.5 I_{10}\left(1-2 \sin \left(\theta_{2}+\theta_{3}\right) \sin \left(\theta_{2}+\theta_{3}\right)\right) \\
c_{31} & =-c_{23}=I_{12} \sin \left(\theta_{3}\right)-I_{5} \cos \left(\theta_{3}\right)-I_{16} \cos \left(\theta_{3}\right)
\end{aligned} \\
& \begin{array}{r}
c_{32}=\sin \left(\theta_{2}+\theta_{3}\right) \cos \left(\theta_{2}+\theta_{3}\right)-I_{15} 2 \sin \left(\theta_{2}+\theta_{3}\right) \cos \left(\theta_{2}+\theta_{3}\right) \\
-I_{16} \cos \left(\theta_{2}\right) \cos \left(\theta_{2}+\theta_{3}\right)-I_{22} \cos \left(\theta_{2}+\theta_{3}\right) \cos \left(\theta_{2}+\theta_{3}\right) \\
{\left[C(q) \cdot \dot{q}^{2}\right]_{6 \times 1}=\left[\begin{array}{l}
c_{12} \cdot q_{2}{ }^{2}+c_{13} \cdot q_{3}{ }^{2} \\
c_{21} \cdot q_{1}{ }^{2}+c_{23} \cdot q_{3}{ }^{2} \\
c_{13} \cdot q_{1}{ }^{2}+c_{32} \cdot q_{2}{ }_{2} \\
0
\end{array}\right]}
\end{array}
\end{aligned}
$$

Gravity $(G)$ Matrix elements are;

$$
[G(q)]_{4 \times 1}=\left[\begin{array}{c}
0 \\
G_{2} \\
G_{3} \\
0
\end{array}\right]
$$

Where,

$$
\begin{gathered}
G_{2}=g_{1} \cos \left(\theta_{2}\right)+g_{2} \sin \left(\theta_{2}+\theta_{3}\right)+g_{3} \sin \left(\theta_{2}\right)+g_{4} \cos \left(\theta_{2}+\theta_{3}\right)+g_{5} \sin \left(\theta_{2}+\theta_{3}\right) \\
G_{3}=g_{2} \sin \left(\theta_{2}+\theta_{3}\right)+g_{4} \cos \left(\theta_{2}+\theta_{3}\right)+g_{5} \sin \left(\theta_{2}+\theta_{3}\right) \\
\text { If }[I]_{4 \times 1}=[B]_{4 \times 1}+[C]_{4 \times 1}+[G]_{4 \times 1}
\end{gathered}
$$

Then $\ddot{q}$ is written as follows;

$$
[\ddot{q}]_{4 \times 1}=\left[A^{-1}(q)\right]_{4 \times 4} \times\left\{[\tau]_{4 \times 1}-[I]_{4 \times 1}\right\}
$$

$K$ is presented as follows;

$$
\begin{gathered}
{[K]_{4 \times 1}=\left\{[\tau]_{4 \times 1}-[I]_{4 \times 1}\right\}} \\
{[\ddot{q}]_{4 \times 1}=\left[A^{-1}(q)\right]_{4 \times 4} \times[K]_{4 \times 1}} \\
{[q]_{4 \times 1}=\iint\left[A^{-1}(q)\right]_{4 \times 4} \times[K]_{4 \times 1}}
\end{gathered}
$$

Basic information about inertial and gravitational constants is show in tables 1 and 2 
Table 1. Inertial Constant Reference $\left(K g \cdot \mathbf{m}^{2}\right)$

\begin{tabular}{|c|c|}
\hline$I_{1}=1.43 \pm 0.05$ & $I_{2}=1.75 \pm 0.07$ \\
\hline$I_{3}=1.38 \pm 0.05$ & $I_{4}=0.69 \pm 0.02$ \\
\hline$I_{5}=0.372 \pm 0.031$ & $I_{6}=0.333 \pm 0.016$ \\
\hline$I_{7}=0.298 \pm 0.029$ & $I_{8}=-0.134 \pm 0.014$ \\
\hline$I_{9}=0.0238 \pm 0.012$ & $I_{10}=-0.0213 \pm 0.0022$ \\
\hline$I_{11}=-0.0142 \pm 0.0070$ & $I_{12}=-0.011 \pm 0.0011$ \\
\hline$I_{13}=-0.00379 \pm 0.0009$ & $I_{14}=0.00164 \pm 0.000070$ \\
\hline$I_{15}=0.00125 \pm 0.0003$ & $I_{16}=0.00124 \pm 0.0003$ \\
\hline$I_{17}=0.000642 \pm 0.0003$ & $I_{18}=0.000431 \pm 0.00013$ \\
\hline$I_{19}=0.0003 \pm 0.0014$ & $I_{20}=-0.000202 \pm 0.0008$ \\
\hline$I_{21}=-0.0001 \pm 0.0006$ & $I_{22}=-0.000058 \pm 0.000015$ \\
\hline$I_{23}=0.00004 \pm 0.00002$ & $I_{m 1}=1.14 \pm 0.27$ \\
\hline$I_{m 2}=4.71 \pm 0.54$ & $I_{m 3}=0.827 \pm 0.093$ \\
\hline$I_{m 4}=0.2 \pm 0.016$ & $I_{m 5}=0.179 \pm 0.014$ \\
\hline$I_{m 6}=0.193 \pm 0.016$ & \\
\hline
\end{tabular}

Table 2. Gravitational Constant (N.m)

\begin{tabular}{|c|c|}
\hline$g_{1}=-37.2 \pm 0.5$ & $g_{2}=-8.44 \pm 0.20$ \\
\hline$g_{3}=1.02 \pm 0.50$ & $g_{4}=0.249 \pm 0.025$ \\
\hline$g_{5}=-0.0282 \pm 0.0056$ & \\
\hline
\end{tabular}

Figure 1 shows the simscape modeling of 4 DOF robot manipulator. 


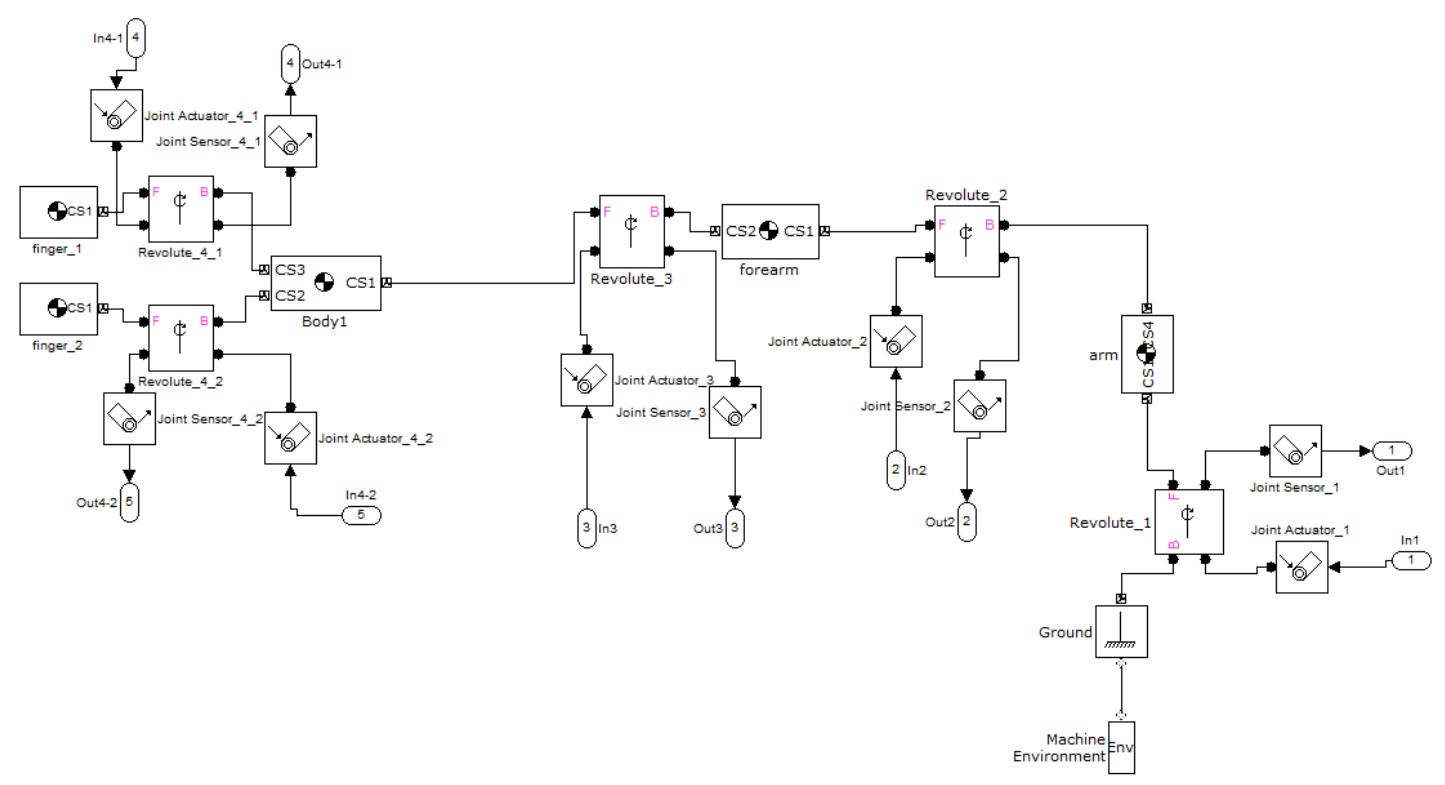

Figure 1. System's Model

System's physical model shows as follow:

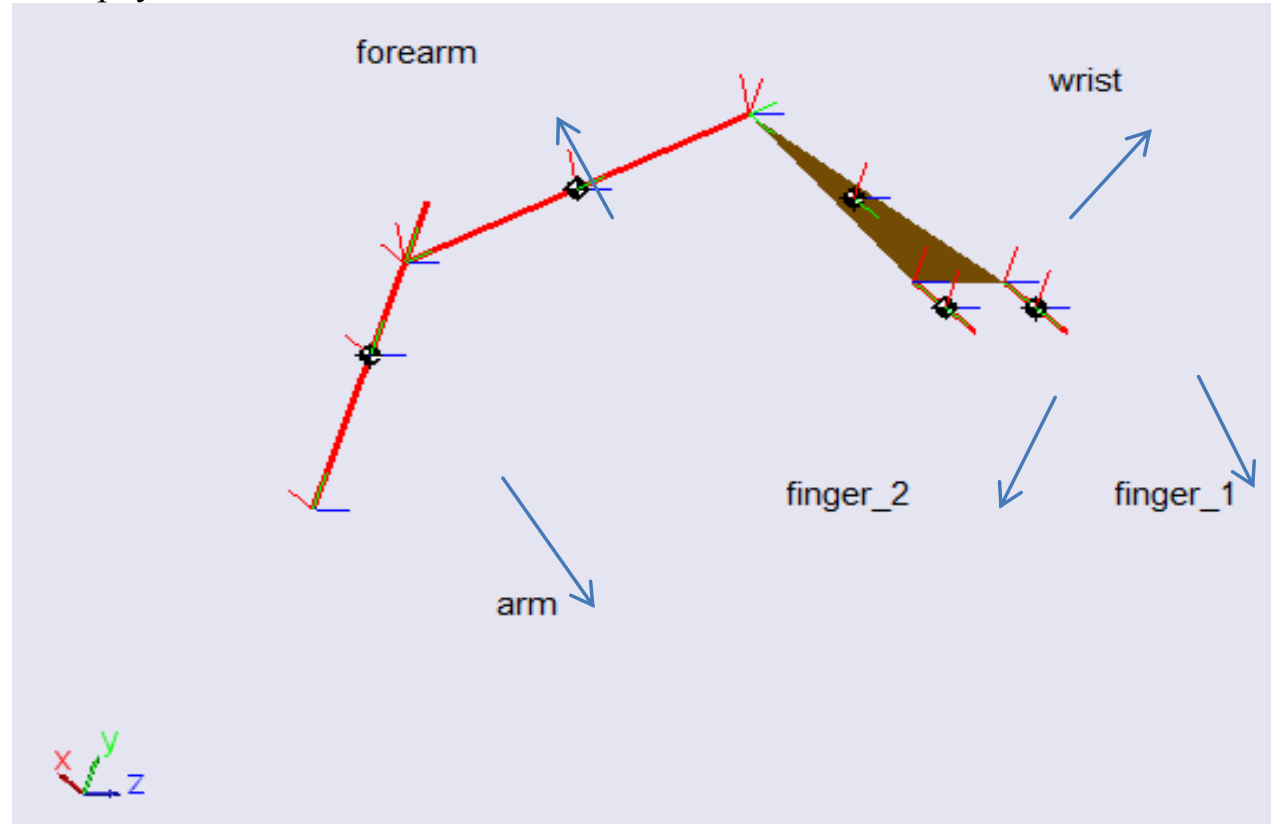

Figure 2. System's Physical Model

\section{Traditional Sliding Mode Control}

Control part is used to adjust the timing between the subparts of robot manipulator to reach the best trajectory. Sliding mode controller (SMC) is a powerful nonlinear controller which has been analyzed by many researchers especially in recent years. This theory was first proposed in the early 1950 by Emelyanov and several co-workers and has been extensively developed since then with the invention of high speed control devices [2]. The main reason to opt for this controller is its acceptable control performance in wide range and solves two most important challenging topics in control which names, stability and robustness. Sliding mode controller is divided into two main sub controllers: discontinues controller $\left(\boldsymbol{\tau}_{\text {dis }}\right)$ and equivalent controller $\left(\boldsymbol{\tau}_{\boldsymbol{e q}}\right)$. Discontinues controller 
causes an acceptable tracking performance at the expense of very fast switching. Conversely in this theory good trajectory following is based on fast switching, fast switching is caused to have system instability and chattering phenomenon. Chattering phenomenon can causes some problems such as saturation and heats the mechanical parts of robot manipulators or drivers. To reduce or eliminate the chattering, various papers have been reported by many researchers which classified into two most important methods: boundary layer saturation method and estimated uncertainties method. In recent years, artificial intelligence theory has been used in nonlinear control systems. Neural network, fuzzy logic and neuro-fuzzy are synergically combined with nonlinear classical controller and used in nonlinear, time variant and uncertain plant. Consider a nonlinear single input dynamic system is defined by [3-5]:

$$
x^{(n)}=f(\vec{x})+b(\vec{x}) u
$$

Where $\mathrm{u}$ is the vector of control input, $\boldsymbol{x}^{(\boldsymbol{n})}$ is the $\boldsymbol{n}^{\text {th }}$ derivation of $\boldsymbol{x}$, $\boldsymbol{x}=\left[\boldsymbol{x}, \dot{\boldsymbol{x}}, \ddot{\boldsymbol{x}}, \ldots, \boldsymbol{x}^{(\boldsymbol{n}-\mathbf{1})}\right]^{\boldsymbol{T}}$ is the state vector, $\boldsymbol{f}(\boldsymbol{x})$ is unknown or uncertainty, and $\boldsymbol{b}(\boldsymbol{x})$ is of known sign function. The main goal to design this controller is train to the desired state; $\boldsymbol{x}_{\boldsymbol{d}}=\left[\boldsymbol{x}_{\boldsymbol{d}}, \dot{\boldsymbol{x}}_{\boldsymbol{d}}, \ddot{\boldsymbol{x}}_{\boldsymbol{d}}, \ldots, \boldsymbol{x}_{\boldsymbol{d}}^{(\boldsymbol{n}-\mathbf{1})}\right]^{\boldsymbol{T}}$, and trucking error vector is defined by[6-8]:

$$
\tilde{x}=x-x_{d}=\left[\tilde{x}, \ldots, \tilde{x}^{(n-1)}\right]^{T}
$$

A time-varying sliding surface $\boldsymbol{s}(\boldsymbol{x}, \boldsymbol{t})$ in the state space $\boldsymbol{R}^{\boldsymbol{n}}$ is given by:

$$
s(x, t)=\left(\frac{d}{d t}+\lambda\right)^{n-1} \tilde{x}=0
$$

$\lambda$ is the positive constant. To further penalize tracking error, integral part can be used in sliding surface part as follows:

$$
s(x, t)=\left(\frac{d}{d t}+\lambda\right)^{n-1}\left(\int_{0}^{t} \tilde{x} d t\right)=0
$$

The main target in this methodology is kept the sliding surface slope $\boldsymbol{s}(\boldsymbol{x}, \boldsymbol{t})$ near to the zero. Therefore, one of the common strategies is to find input $\boldsymbol{U}$ outside of $\boldsymbol{s}(\boldsymbol{x}, \boldsymbol{t})$.

$$
\frac{1}{2} \frac{d}{d t} s^{2}(x, t) \leq-\zeta|s(x, t)|
$$

$\zeta$ is positive constant.

$$
\text { If } S(0)>0 \rightarrow \frac{d}{d t} S(t) \leq-\zeta
$$

To eliminate the derivative term, it is used an integral term from $t=0$ to $t=t_{\text {reach }}$

$$
\int_{t=0}^{t=t_{\text {reach }}} \frac{d}{d t} S(t) \leq-\int_{t=0}^{t=t_{\text {reach }}} \eta \rightarrow S\left(t_{\text {reach }}\right)-S(0) \leq-\zeta\left(t_{\text {reach }}-0\right)
$$

Where $t_{\text {reach }}$ is the time that trajectories reach to the sliding surface so, suppose $\mathrm{S}\left(t_{\text {reach }}=0\right)$ defined as;

$$
0-S(0) \leq-\eta\left(t_{\text {reach }}\right) \rightarrow t_{\text {reach }} \leq \frac{S(0)}{\zeta}
$$


if $S(0)<0 \rightarrow 0-S(0) \leq-\eta\left(t_{\text {reach }}\right) \rightarrow S(0) \leq-\zeta\left(t_{\text {reach }}\right) \rightarrow t_{\text {reach }} \leq \frac{|S(0)|}{\eta}$

Above equation guarantees time to reach the sliding surface is smaller than $\frac{|\boldsymbol{S}(\mathbf{0})|}{\zeta}$ since the trajectories are outside of $S(t)$.

$$
\text { if } S_{t_{\text {reach }}}=S(0) \rightarrow \operatorname{error}\left(x-x_{d}\right)=0
$$

suppose $\mathrm{S}$ is defined as

$$
s(x, t)=\left(\frac{d}{d t}+\lambda\right) \quad \tilde{x}=\left(\dot{\mathrm{x}}-\dot{\mathrm{x}}_{\mathrm{d}}\right)+\lambda\left(\mathrm{x}-\mathrm{x}_{\mathrm{d}}\right)
$$

The derivation of S, namely, $\dot{S}$ can be calculated as the following;

$$
\dot{S}=\left(\ddot{\mathrm{x}}-\ddot{\mathrm{x}}_{\mathrm{d}}\right)+\lambda\left(\dot{\mathrm{x}}-\dot{\mathrm{x}}_{\mathrm{d}}\right)
$$

suppose the second order system is defined as;

$$
\ddot{x}=f+u \rightarrow \dot{S}=f+U-\ddot{x}_{d}+\lambda\left(\dot{\mathrm{x}}-\dot{\mathrm{x}}_{\mathrm{d}}\right)
$$

$\boldsymbol{f}$ is the dynamic uncertain, and also since $S=0$ and $\dot{S}=0$, to have the best approximation, $\widehat{\boldsymbol{U}}$ is defined as

$$
\widehat{U}=-\hat{f}+\ddot{x}_{d}-\lambda\left(\dot{\mathrm{x}}-\dot{\mathrm{x}}_{\mathrm{d}}\right)
$$

A simple solution to get the sliding condition when the dynamic parameters have uncertainty is the switching control law:

$$
U_{d i s}=\widehat{U}-K(\vec{x}, t) \cdot \operatorname{sgn}(s)
$$

The switching function $\operatorname{sgn}(\mathrm{S})$ is defined as

$$
\operatorname{sgn}(s)= \begin{cases}1 & s>0 \\ -1 & s<0 \\ 0 & s=0\end{cases}
$$

The $\boldsymbol{K}(\overrightarrow{\boldsymbol{x}}, \boldsymbol{t})$ is the positive constant.

$$
\frac{1}{2} \frac{d}{d t} s^{2}(x, t)=\dot{S} \cdot S=[f-\hat{f}-K \operatorname{sgn}(s)] \cdot S=(f-\hat{f}) \cdot S-K|S|
$$

The sliding surface can be calculated as

$$
s(x, t)=\left(\frac{d}{d t}+\lambda\right)^{2}\left(\int_{0}^{t} \tilde{x} d t\right)=\left(\dot{\mathrm{x}}-\dot{\mathrm{x}}_{\mathrm{d}}\right)+2 \lambda\left(\dot{\mathrm{x}}-\dot{\mathrm{x}}_{\mathrm{d}}\right)-\lambda^{2}\left(\mathrm{x}-\mathrm{x}_{\mathrm{d}}\right)
$$

in this method the approximation of $\boldsymbol{U}$ is computed as

$$
\widehat{U}=-\hat{f}+\ddot{x}_{d}-2 \lambda\left(\dot{\mathrm{x}}-\dot{\mathrm{x}}_{\mathrm{d}}\right)+\lambda^{2}\left(\mathrm{x}-\mathrm{x}_{\mathrm{d}}\right)
$$

The sliding mode control law for multi degrees of freedom robot manipulator is written as:

$$
\tau=\tau_{e q}+\tau_{d i s}
$$


The model-based component $\boldsymbol{\tau}_{\boldsymbol{e q}}$ is the nominal dynamics of systems calculated as follows:

$\boldsymbol{\tau}_{\text {dis }}$ is computed as;

$$
\tau_{e q}=\left[D^{-1}(f+C+G)+\dot{S}\right] D
$$

$$
\tau_{\text {dis }}=K \cdot \operatorname{sgn}(S)
$$

The sliding mode control of robot manipulator is calculated as;

$$
\tau=\left[D^{-1}(f+C+G)+\dot{S}\right] D+K \cdot \operatorname{sgn}(S)
$$

Figure 3 shows trajectory following in traditional SMC. Regarding to this Figure it has high frequency oscillation but in rise time point of view, it has an acceptable performance and it has about 0.6 second delay time.

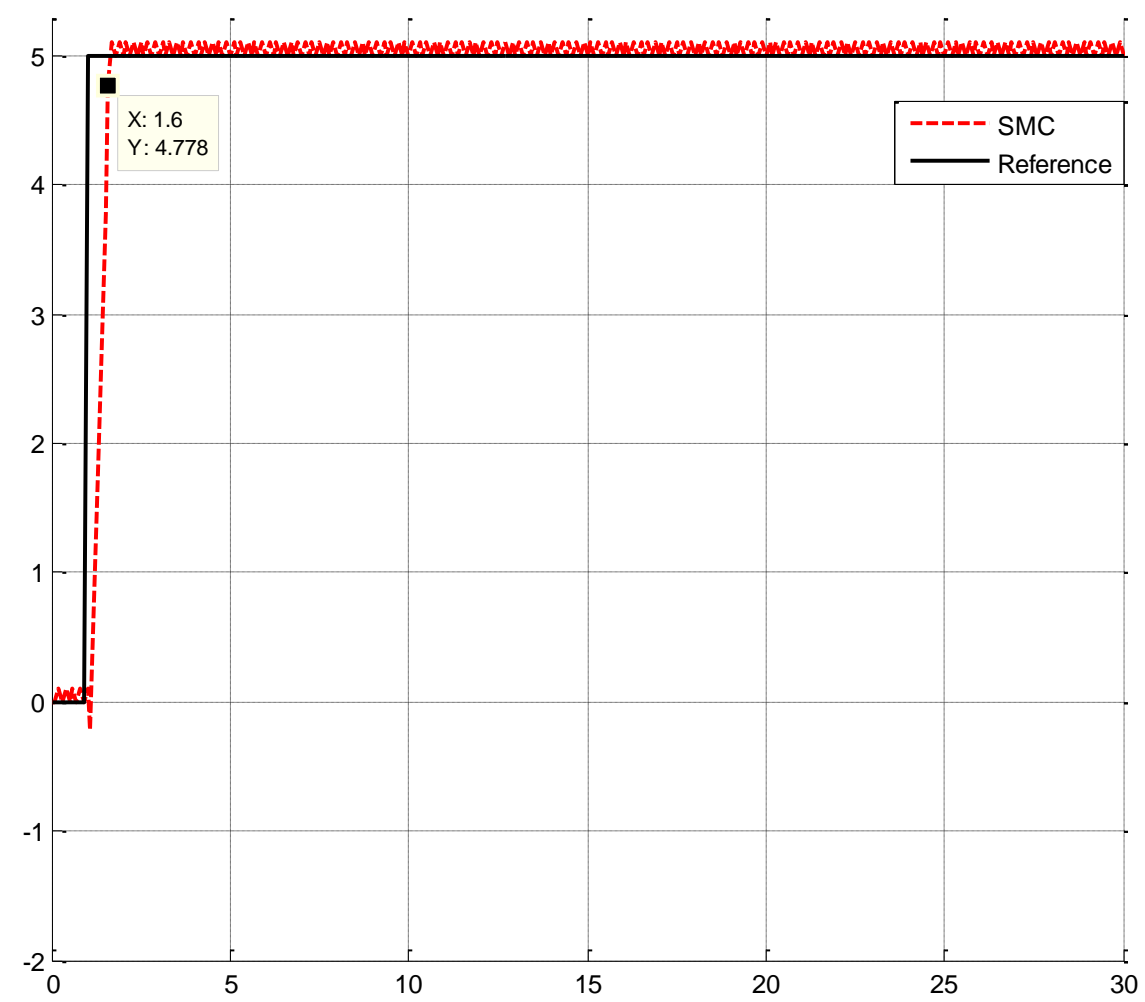

Figure 3. SMC Trajectory Performance

Figure 4 shows the torque performance. According to this Figure, SMC has extremely high frequency oscillation. 


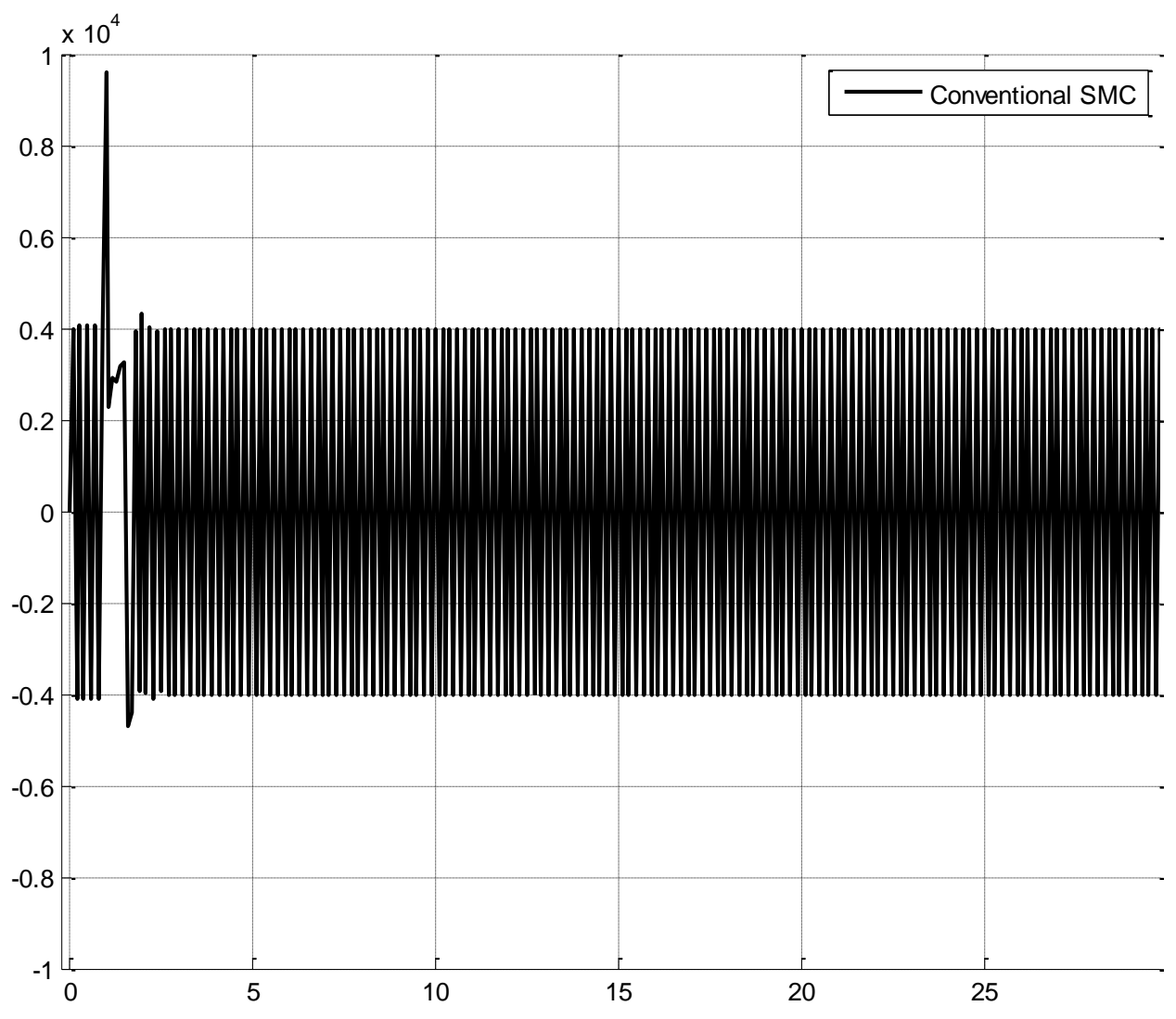

Figure 4. SMC Torque Performance

Figure 5 shows the power of disturbance rejection. According to the following Figure this algorithm is extremely robust and stable in presence of uncertainty. 


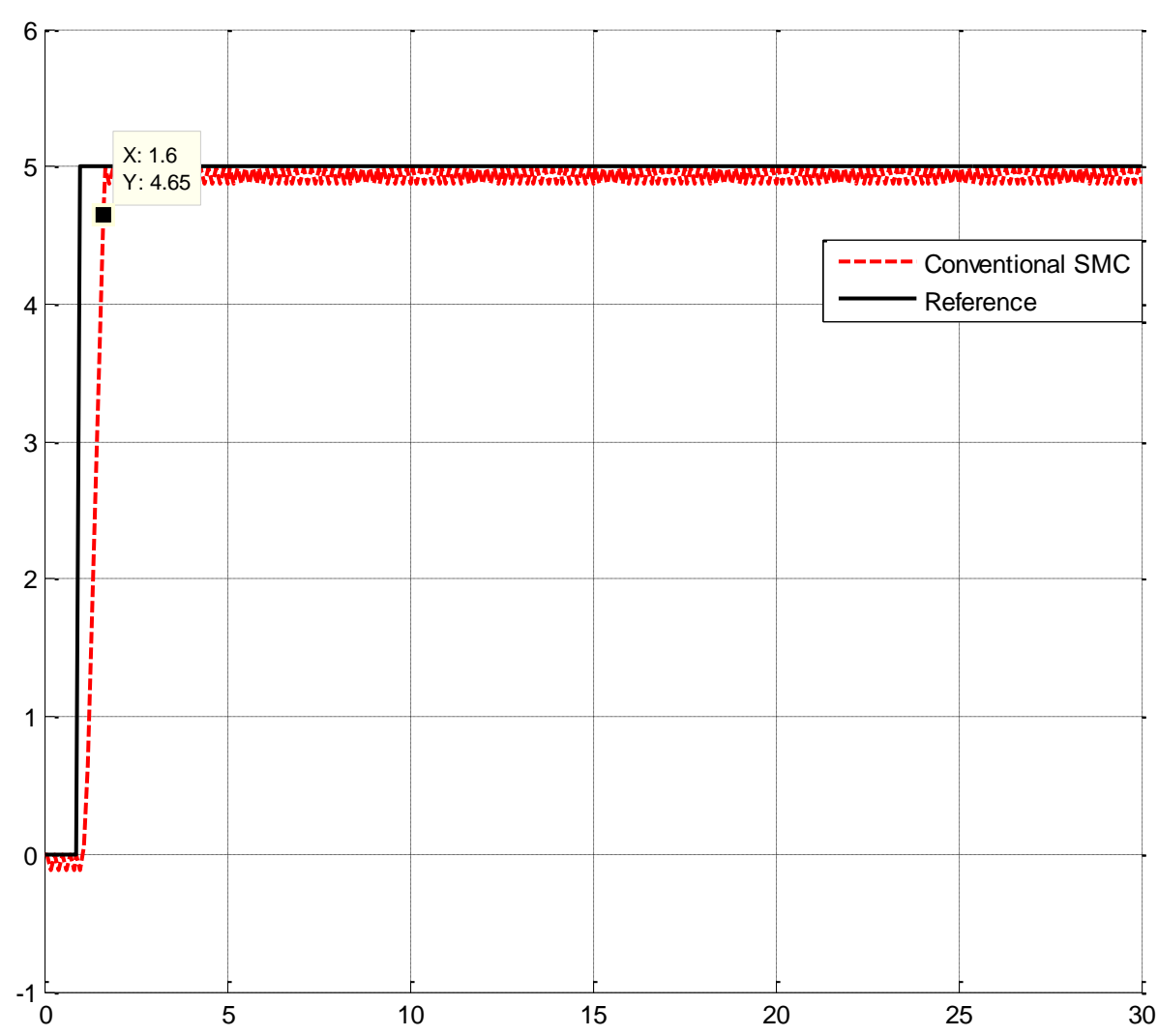

Figure 5. Power of Disturbance Rejection in SMC

\section{Design Artificial Intelligence based Surface Slope}

In a typical PD method, the controller corrects the error between the desired input value and the measured value. Since the actual position is the measured signal. The derivative part of PD methodology is worked based on change of error and the derivative coefficient. In this research the modified PD is used based on boundary derivative part.

$$
\begin{gathered}
\dot{e}(t) \triangleq\left(\frac{S}{\alpha S+\beta}\right) \times e(t) \\
U_{P D}=K_{p_{a}} e+K_{V_{a}} \dot{e}
\end{gathered}
$$

The result of modified PD method shows the power of disturbance rejection in this methodology. Based on the modified formulation the partly linear sliding mode controller formulation is;

$$
\tau_{M-d i s}=K \cdot \operatorname{sgn}(\lambda e+\dot{e})=K \cdot \operatorname{sgn}\left(\lambda e+\left(\frac{S}{\alpha S+\beta}\right) \times e(t)\right)
$$

In the proposed method fuzzy rule base was designed to have a nonlinear sliding surface slope function. The fuzzy system can be defined as below

$$
\begin{gathered}
f(x)=\tau_{\text {fuzzy }}=\sum_{l=1}^{M} \theta^{T} \zeta(x)=\psi(e, \dot{e}) \\
\theta=\left(\theta^{1}, \theta^{2}, \theta^{3}, \ldots \ldots, \theta^{M}\right)^{T}, \zeta(x)=\left(\zeta^{1}(x), \zeta^{2}(x), \zeta^{3}(x), \ldots \ldots, \zeta^{M}(x)\right)^{T}
\end{gathered}
$$




$$
\zeta^{1}(x)=\frac{\sum_{i} \mu_{(x i) x_{i}}}{\sum_{i} \mu_{(x i)}}
$$

$\theta=\left(\theta^{1}, \theta^{2}, \theta^{3}, \ldots \ldots, \theta^{M}\right)$ is adjustable parameter and $\mu_{(x i)}$ is membership function. Error base fuzzy controller can be defined as

$$
\tau_{f u z z y}=\psi(e, \dot{e})
$$

To eliminate the chattering fuzzy inference system is used instead of saturation and/or switching function.

$$
\text { if } S=0 \text { then }-\dot{e}=\lambda e
$$

The fuzzy division can be reached the best state when $S . \dot{S}<0$ and the error is minimum by the following formulation

$$
\theta^{*}=\arg \min \left[\operatorname{Sup}_{x \in U}\left|\sum_{l=1}^{M} \theta^{T} \zeta(x)-\tau_{e q u}\right|\right]
$$

$\theta^{*}$ is the minimum error, $\sup _{x \in U}\left|\sum_{l=1}^{M} \theta^{T} \zeta(x)-\tau_{\text {equ }}\right|$ is the minimum approximation error. Figure 6 shows the fuzzy instead of saturation function.

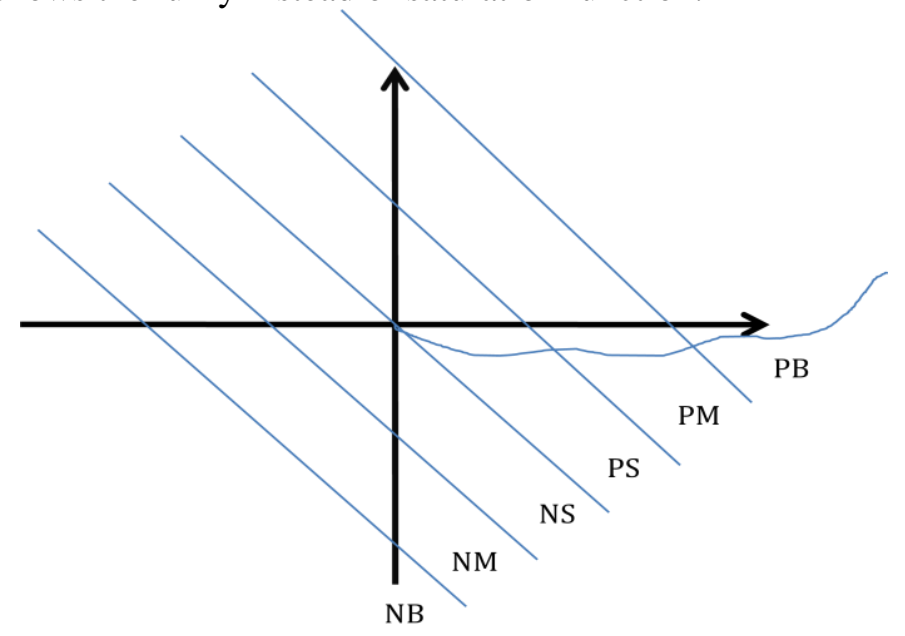

Figure 6. Nonlinear Fuzzy Inference System instead of Saturation Function

To estimate the dynamic formulation in uncertainty fuzzy logic method is applied to equivalent part.

$$
\tau=K \cdot \operatorname{sgn}\left(\lambda e+\left(\frac{S}{0.1 S+1}\right) \times e(t)\right)+\tau_{f u z z y}
$$

Based on fuzzy logic methodology

$$
f(x)=\tau_{f u z z y}=\sum_{l=1}^{M} \theta^{T} \zeta(x)
$$

$\boldsymbol{\theta}^{\boldsymbol{T}}$ is adjustable parameter (gain updating factor) and $\boldsymbol{\zeta}(\boldsymbol{x})$ is defined by;

$$
\zeta(x)=\frac{\sum_{i} \mu\left(x_{i}\right) x_{i}}{\sum_{i} \mu\left(x_{i}\right)}
$$


Figure 7 shows the trajectory following in this algorithm. According to this Figure, the high frequency oscillation eliminates but the time response increases from 0.6 seconds in conventional SMC to 1.4 seconds in proposed methodology.

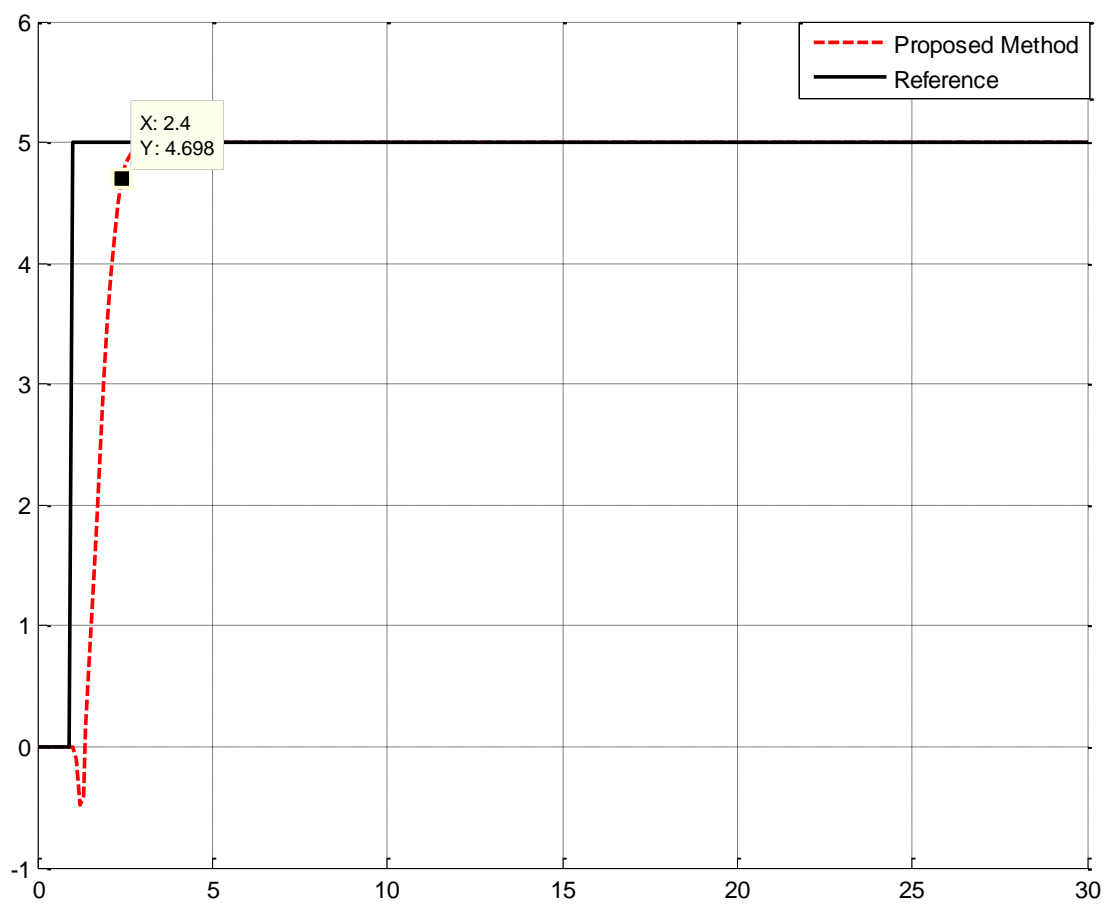

Figure 7. Trajectory Following in Proposed Surface Slope SMC

Figure 8 shows torque performance in proposed method.

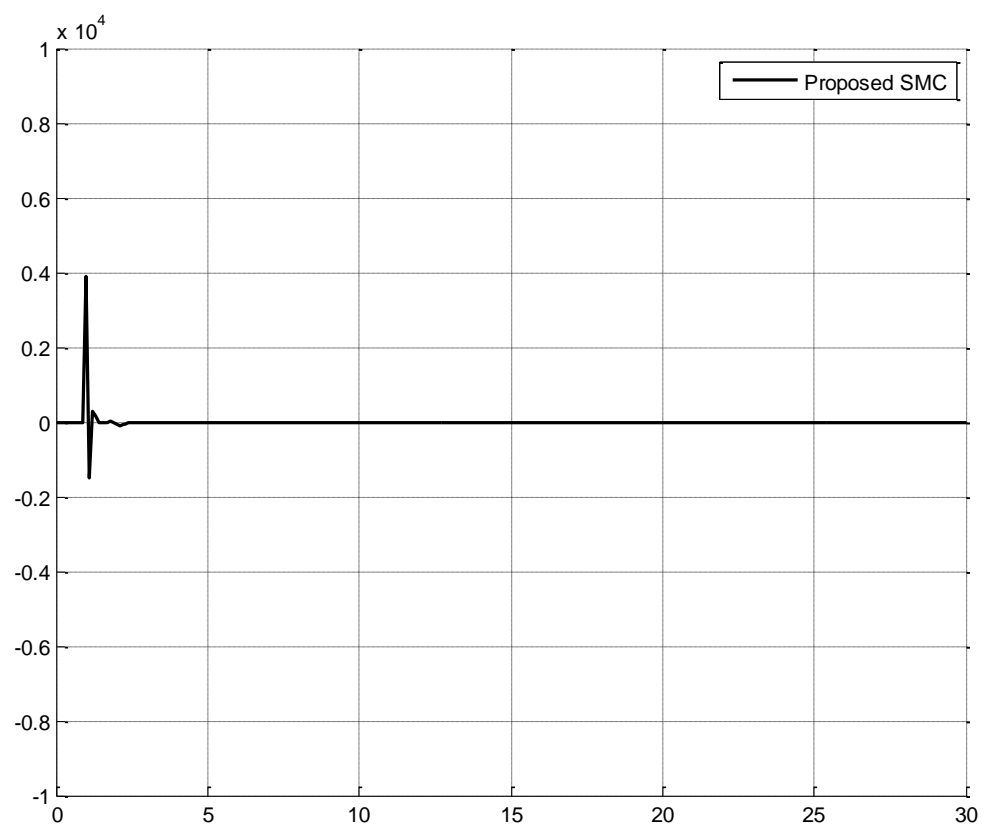

Figure 8. Torque Performance in Proposed Surface Slope SMC

Based on Figure 8, this method reduces the rate of energy consumed because of chattering elimination. 
Figure 9 shows the power of disturbance rejection. This method is more robust than pure SMC as well as saturation SMC. According to Figure 9, the rise time in this algorithm fixed in 1.4 seconds. Therefore, these methods can support the time stability.

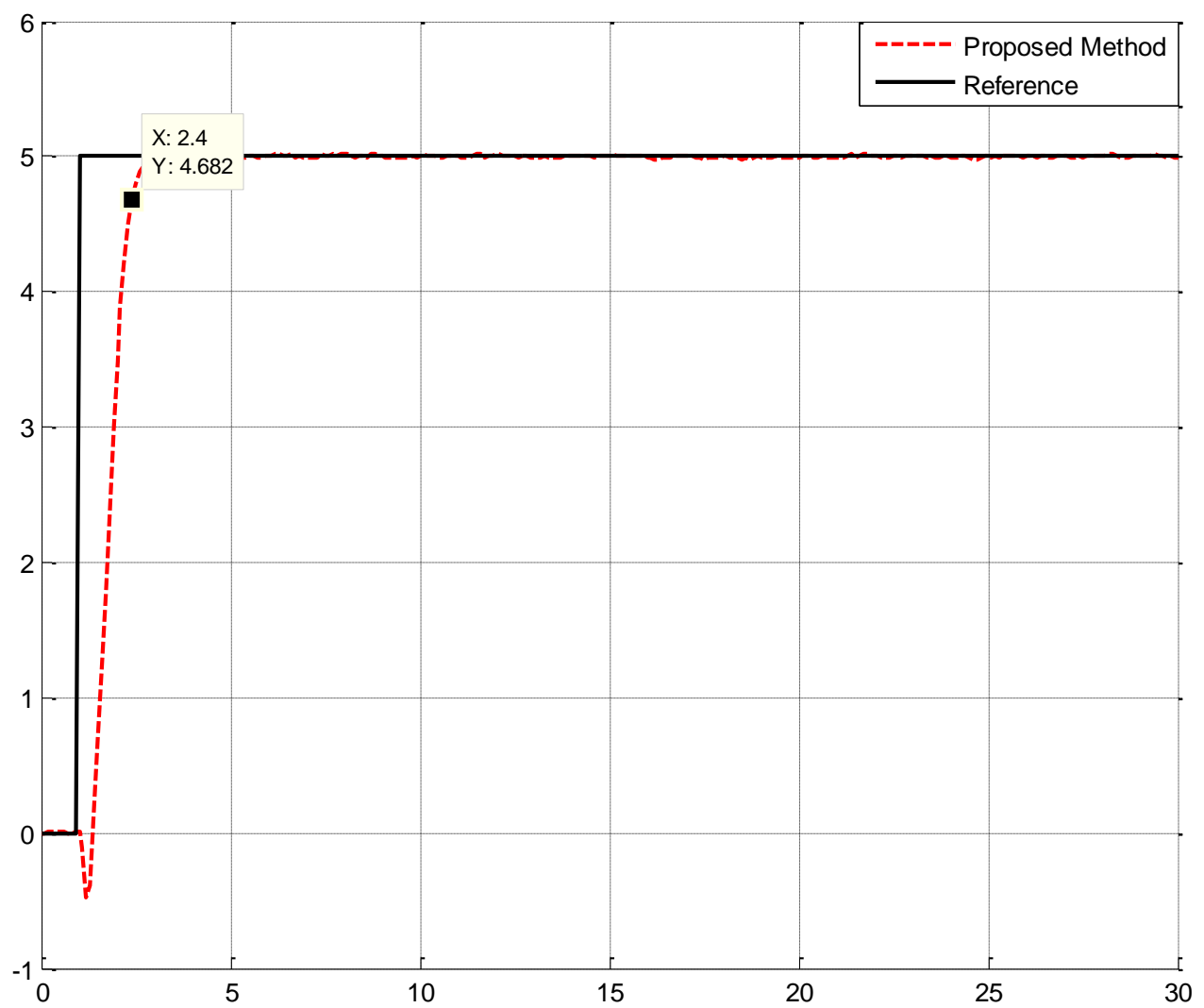

Figure 9. Power of Disturbance Rejection in Proposed Surface Slope SMC

\section{Conclusion}

Refer to the research, a chattering free fuzzy sliding mode controller is introduced and this suitability for use in the control of medical robot manipulator has proposed. Sliding mode control methodology is selected as the main controller to construct the control law and address the stability and robustness of the close-loop system. The proposed approach effectively combines the design techniques from conventional switching surface slope sliding mode control and fuzzy logic to improve the performance of sliding surface slope and improve the trajectory following, disturbance rejection and eliminate the chattering phenomenon, and enhance the robustness property of the controller. The system performance in sliding mode controller is sensitive to the sliding function. Therefore, compute the optimum value of sliding function for a system is important which this problem has solved by adjusting the surface slope of the sliding function continuously in real-time. The chattering phenomenon is estimated by the fuzzy method when estimating the saturation/switching function with rule bases. This controller solved chattering phenomenon by applied modified fuzzy inference system method in new sliding mode controller.

\section{Acknowledgment}

This work was supported by the Iranian Institute of Advance Science and Technology Program of Iran under grant no. 2015-Persian Gulf-2. 


\section{Project Title: Research on Intelligent FPGA-Based Algorithm for Four Degrees of Freedom Joints}

Iranian center of Advance Science and Technology (IRAN SSP) is one of the independent research centers specializing in research and training across of Control and Automation, Electrical and Electronic Engineering, and Mechatronics \& Robotics in Iran. At IRAN SSP research center, we are united and energized by one mission to discover and develop innovative engineering methodology that solve the most important challenges in field of advance science and technology. The IRAN SSP Center is instead to fill a long standing void in applied engineering by linking the training a development function one side and policy research on the other. This center divided into two main units:

- $\quad$ Education unit

- $\quad$ Research and Development unit

\section{Please follow IRANSSP research and training group: http://iranssp.org/english/}

\section{References}

[1] B. Siciliano and O. Khatib, "Springer handbook of robotics", Springer Science \& Business Media, (2008).

[2] V. Utkin, "Variable structure systems with sliding modes", IEEE Transactions on Automatic Control, vol. 22, no. 2, (2002), pp. 212-222.

[3] R. A. DeCarlo, S. H. Zak and G. P. Matthews, "Variable structure control of nonlinear multivariable systems: a tutorial", Proceedings of the IEEE, vol. 76, no. 3, (2002), pp. 212-232.

[4] B. Wu, Y. Dong, S. Wu, D. Xu and K. Zhao, "An integral variable structure controller with fuzzy tuning design for electro-hydraulic driving Stewart platform", $1^{\text {st }}$ International Symposium on Systems and Control in Aerospace and Astronautics, pp. 940-945, (2006).

[5] H. Elmali and N. Olgac, "Implementation of sliding mode control with perturbation estimation (SMCPE)”, IEEE Transactions on Control Systems Technology, vol. 4, no. 1, (2002), pp. 79-85.

[6] J. J. Slotine and S. Sastry, "Tracking control of non-linear systems using sliding surfaces, with application to robot manipulators", International Journal of Control, vol. 38, no. 2, (1983), pp. 465-492.

[7] C. C. Weng and W. S. Yu, "Adaptive fuzzy sliding mode control for linear time-varying uncertain systems", IEEE International conference on Fuzzy Systems, (2008), pp. 1483-1490.

[8] Y. C. Hsu and H. A. Malki, "Fuzzy variable structure control for MIMO systems", IEEE International conference on Fuzzy Systems, (2002), pp. 280-285.

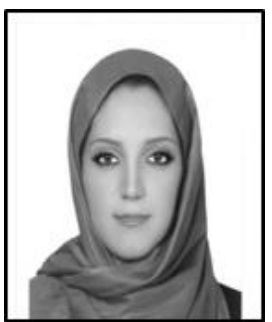

\section{Authors}

Somayeh Jowkar, he currently is research assistant at Institute of Advanced Science and Technology, Research Center, IRAN SSP. She is research assistant of team to Design Intelligent FPGABased Control Unit to Control of 4-DOF Medical Robot Manipulator since July, 2015 to now, research student (21 researchers) to design high precision and fast dynamic controller for multi-degrees of freedom actuator since 2014 to date, and published 2 journal papers since 2014 to date. Her current research interests are nonlinear control, artificial control system, Microelectronic Device and HDL design. 


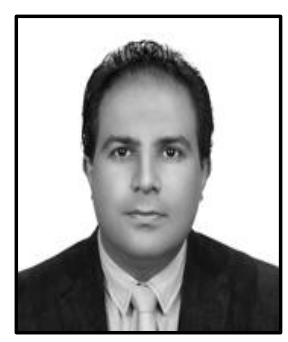

Farzin Piltan, he is an outstanding scientist in the field of Electronics and Control engineering with expertise in the areas of nonlinear systems, robotics, and microelectronic control. Mr. Piltan is an advanced degree holder in his field. Currently, Mr. Piltan is the Head of Mechatronics, Intelligent System, and Robotics Laboratory at the Iranian Institute of Advanced Science and Technology (IRAN SSP). Mr. Piltan led several high impact projects involving more than 150 researchers from countries around the world including Iran, Finland, Italy, Germany, South Korea, Australia, and the United States. Mr. Piltan has authored or coauthored more than 140 papers in academic journals, conference papers and book chapters. His papers have been cited at least 3900 times by independent and dependent researchers from around the world including Iran, Algeria, Pakistan, India, China, Malaysia, Egypt, Columbia, Canada, United Kingdom, Turkey, Taiwan, Japan, South Korea, Italy, France, Thailand, Brazil and more. Moreover, Mr. Piltan has peer-reviewed at least 23 manuscripts for respected international journals in his field. Mr. Piltan will also serve as a technical committee member of the upcoming EECSI 2015 Conference in Indonesia. Mr. Piltan has served as an editorial board member or journal reviewer of several international journals in his field as follows: International Journal of Control And Automation (IJCA), Australia, ISSN: 2005-4297, International Journal of Intelligent System and Applications (IJISA), Hong Kong, ISSN:2074-9058, IAES International Journal Of Robotics And Automation, Malaysia, ISSN:2089-4856, International Journal of Reconfigurable and Embedded Systems, Malaysia, ISSN:20894864.

Mr. Piltan has acquired a formidable repertoire of knowledge and skills and established himself as one of the leading young scientists in his field. Specifically, he has accrued expertise in the design and implementation of intelligent controls in nonlinear systems. Mr. Piltan has employed his remarkable expertise in these areas to make outstanding contributions as detailed follows:Nonlinear control for industrial robot manipulator (2010IRAN SSP), Intelligent Tuning The Rate Of Fuel Ratio In Internal Combustion Engine (2011-IRANSSP), Design High Precision and Fast Dynamic Controller For Multi-Degrees Of Freedom Actuator (2013-IRANSSP), Research on Full Digital Control for Nonlinear Systems (2011-IRANSSP), Micro-Electronic Based Intelligent Nonlinear Controller (2015-IRANSSP), Active Robot Controller for Dental Automation (2015-IRANSSP), Design a Micro-Electronic Based Nonlinear Controller for First Order Delay System (2015-IRANSSP).

The above original accomplishments clearly demonstrate that Mr. Piltan has performed original research and that he has gained a distinguished reputation as an outstanding scientist in the field of electronics and control engineering. Mr. Piltan has a tremendous and unique set of skills, knowledge and background for his current and future work. He possesses a rare combination of academic knowledge and practical skills that are highly valuable for his work. In 2011, he published 28 first author papers, which constitute about $30 \%$ of papers published by the Department of Electrical and 


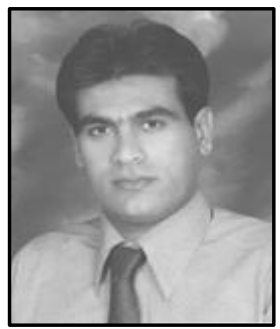

Electronic Engineering at University Putra Malaysia. Additionally, his 28 papers represent about $6.25 \%$ and $4.13 \%$ of all control and system papers published in Malaysia and Iran, respectively, in 2011.

Amirzubir Sahamijoo, he iscurrently is senior research assistant at Institute of Advanced Science and Technology, Research Center, IRAN SSP. He is senior research assistant of team to Design Intelligent FPGA-Based Control Unit to Control of 4-DOF Medical Robot Manipulator since July, 2015 to now, research assistant of team ( 8 researchers) to design a Microelectronic Based nonlinear controller for first order delay system since March, 2015 to now, research student (21 researchers) to design high precision and fast dynamic controller for multi-degrees of freedom actuator since 2014 to date, research student (9 researchers) to design Prevent the Risk of Lung Cancer Progression Based on Fuel Ratio Optimization since 2014 to date, and published 4 journal papers since 2014 to date. His current research interests are nonlinear control, artificial control system, Microelectronic Device, Internal Combustion Engine, and HDL design.

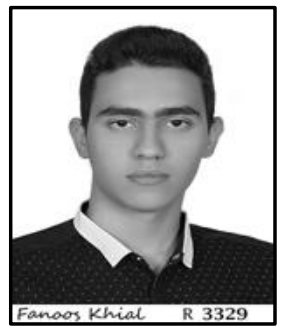

Ali Taghizadegan, he is currently studying as a student in the second grade of Shahid dastgheib's 1 high school and Research Student at Iranian Institute of Advanced Science and Technology, Research and Training Center, IRAN SSP. He is research student of team (6 researchers) to design Micro-electronic Based nonlinear controller for Four Degrees of Freedom Surgical Robot Manipulator since August 2015. His current research interests are nonlinear control, artificial control system, Microelectronic Device, and HDL design.

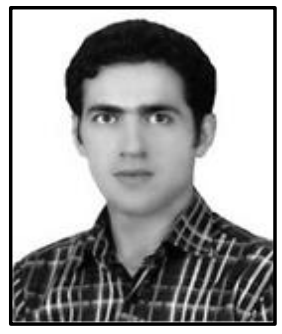

Rouhollah Bahrami, he is currently is research student at Institute of Advanced Science and Technology, Research Center, IRAN SSP. He is research student of team (6 researchers) to design Micro-electronic Based nonlinear controller for Four Degrees of Freedom Surgical Robot Manipulator since August 2015. His current research interests are nonlinear control, artificial control system, Microelectronic Device, and HDL design.

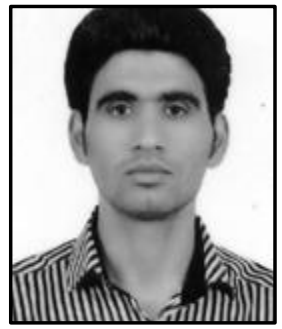

Hossein Rashidi Bod, he currently is research student at Institute of Advanced Science and Technology, Research Center, IRAN SSP. He is research student of team (6 researchers) to design Micro-electronic Based nonlinear controller for Four Degrees of Freedom Surgical Robot Manipulator since August 2015. His current research interests are nonlinear control, artificial control system, Microelectronic Device, and HDL design. 


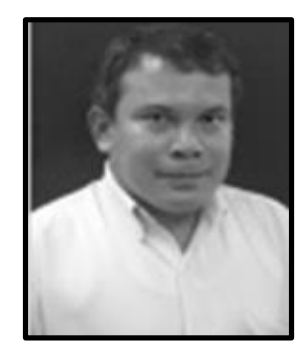

Nasri Sulaiman, he is an advisor and supervisor of several high impact projects involving more than 150 researchers from countries around the world including Iran, Malaysia, Finland, Italy, Germany, South Korea, Australia, and the United States. Dr. Nasri Sulaiman has authored or co-authored more than 80 papers in academic journals, conference papers and book chapters. His papers have been cited at least 3000 times by independent and dependent researchers from around the world including Iran, Algeria, Pakistan, India, China, Malaysia, Egypt, Columbia, Canada, United Kingdom, Turkey, Taiwan, Japan, South Korea, Italy, France, Thailand, Brazil and more. Dr. Nasri Sulaiman has employed his remarkable expertise in these areas to make outstanding contributions as detailed below:

- Design of a reconfigurable Fast Fourier Transform (FFT) Processor using multi-objective Genetic Algorithms (2008-UPM)

- Power consumption investigation in reconfigurable Fast Fourier Transform (FFT) processor (2010-UPM)

- Crest factor reduction and digital predistortion Implementation in Orthogonal Frequency Division multiplexing (ofdm) systems (2011-UPM)

- High Performance Hardware Implementation of a MultiObjective Genetic Algorithm, (RUGS), Grant amount RM42,000.00, September (2012-UPM)

- Nonlinear control for industrial robot manipulator (2010-IRAN SSP)

- Intelligent Tuning The Rate Of Fuel Ratio In Internal Combustion Engine (2011-IRANSSP)

- Design High Precision and Fast Dynamic Controller For MultiDegrees Of Freedom Actuator (2013-IRANSSP)

- Research on Full Digital Control for Nonlinear Systems (2011IRANSSP)

- Micro-Electronic Based Intelligent Nonlinear Controller (2015IRANSSP)

- Active Robot Controller for Dental Automation (2015IRANSSP)

- Design a Micro-Electronic Based Nonlinear Controller for First Order Delay System (2015-IRANSSP) 